\title{
Longitudinal associations between neighbourhood trust, social support and physical activity in adolescents: evidence from the Olympic Regeneration in East London (ORiEL) study
}

\section{Word count: 3774}

Nicolas Berger

Population Health Innovation Lab, Department of Public Health, Environments and Society, London School of Hygiene \& Tropical Medicine, London, United Kingdom, Nicolas.Berger@lshtm.ac.uk

Daniel Lewis

Population Health Innovation Lab, Department of Public Health, Environments and Society, London School of Hygiene \& Tropical Medicine, London, United Kingdom, Daniel.Lewis@Ishtm.ac.uk

Data Science Campus, Office for National Statistics, London, United Kingdom

Matteo Quartagno

MRC Clinical Trials Unit, University College London, London, United Kingdom, m.quartagno@ucl.ac.uk

Department of Medical Statistics, London School of Hygiene \& Tropical Medicine, London, United Kingdom

Edmund Njeru (first name) Njagi (last name)

Department of Non-communicable Disease Epidemiology, London School of Hygiene \& Tropical Medicine, London, United Kingdom, Edmund.Njeru.Njagi@Ishtm.ac.uk

Steven Cummins

Population Health Innovation Lab, Department of Public Health, Environments and Society, London School of Hygiene \& Tropical Medicine, London, United Kingdom, Steven.Cummins@lshtm.ac.uk

Corresponding author:

Nicolas Berger, Department of Public Health, Environments and Society, London School of Hygiene \& Tropical Medicine, Nicolas.Berger@Ishtm.ac.uk, 15-17 Tavistock Place, London WC1H 9SH, UK 


\section{What is already known on this subject?}

- Previous studies suggest that the social environment could contribute to inequalities in physical activity behaviours.

- Social support and social capital (including social cohesion and neighbourhood trust) are positively associated with total and recreational physical activity.

- However, longitudinal evidence on whether changes in social support and social capital are associated with changes in physical activity is still scarce, particularly for young people outside of the USA.

\section{What this study adds?}

- We found that different aspects of the social environment predict different types of physical activity, in a relatively deprived and ethnically diverse UK adolescent population.

Neighbourhood trust was positively associated with leisure-type physical activity, while social support from friends and family was positively associated with walking for leisure. There was some evidence that changes in exposures led to changes in physical activity outcomes (1 year follow-up).

- Policymakers and practitioners should consider tailoring interventions to promote social support and social capital by physical activity type. 


\begin{abstract}
Background

Most UK adolescents do not achieve recommended levels of physical activity (PA). Previous studies suggest that the social environment could contribute to inequalities in PA behaviours, but longitudinal evidence is limited. We examined whether neighbourhood trust and social support were longitudinally associated with four common forms of PA: walking to school, walking for leisure, outdoor PA, and pay and play PA. We further assessed whether gender moderated these associations.
\end{abstract}

\title{
Methods
}

We used longitudinal data from the Olympic Regeneration in East London (ORiEL). In 2012, 3,106 adolescents aged 11-12 were enrolled from 25 schools in four deprived boroughs of East London, UK. Adolescents were followed-up in 2013 and 2014. The final sample includes 2,664 participants interviewed at waves 2 and 3. We estimated logistic regression models using Generalised Estimating Equations (GEE) (pooled models) and proportional odds models (models of change) to assess associations between the social environment exposures and the PA outcomes, adjusting for potential confounders. Item non-response was handled using multi-level multiple imputation.

\section{Results}

We found that different aspects of the social environment predict different types of PA. Neighbourhood trust was positively associated with leisure-type PA. Social support from friends and family was positively associated with walking for leisure. There was some evidence that changes in exposures led to changes in the PA outcomes. Associations did not systematically differ by gender.

\section{Conclusion}

These results confirm the importance of the social environment to predict PA and its change over time in a deprived and ethnically diverse adolescent population.

\section{Keywords}

adolescents; East London; social environment; trust; social support; PA; walking; social capital; social cohesion 


\section{Introduction}

As physical activity (PA) declines during childhood and adolescence [1], the majority of adolescents do not achieve the recommended level of PA in the UK [2]. Increasing and maintaining PA in this group is crucial because adolescence is an important period in the lifecourse during which life-long health behaviours start forming [3]. Among the many potential multi-level determinants of PA, features of the social environment have received particular scrutiny over recent years [4].

The social environment is defined as 'the immediate physical surroundings, social relationships, and cultural milieus within which defined groups of people function and interact' [5]. It encompasses a range of social constructs, among which social capital and social support are prominent tools to understand how the social context affects health [6]. Social capital is defined as the social resources that are accessed by individuals through their membership to a group or a network, including trust, norms of reciprocity and ability to undertake collective action $[4,7]$. Social capital is hypothesised to affect health behaviours through three primary mechanisms: social contagion, collective efficacy and informal social control [4]. The evidence to date in the UK and elsewhere has shown that aspects of social capital - including social cohesion and neighbourhood trust - were positively associated with total and recreational PA in adults and adolescents [8-14].

Social support describes resources provided from interpersonal relationships that can influence behaviour such as PA. These resources are diverse and include: psychological/emotional support (e.g. encouragement, praise), instrumental support (e.g. equipment, transport to a PA facility), coparticipation (e.g. performing an activity with an adolescent), informational support (e.g. providing advice or instructions about an activity), and support as a role model [15]. Parents, family members and friends constitute the main sources of social support for PA in adolescents [16]. The growing literature on the benefits of social support for health behaviours has identified social support as one of the most consistent correlates of PA in young people. During adolescence, transportation, encouragement and role modelling are important types of social support provided by parents, while friends' encouragement and co-participation in activities are the most salient resources of social support provided by friends [16-20].

However, longitudinal evidence on whether changes in social capital and social support are associated with changes in PA is still scarce, in particular in young people and outside of the USA $[19,21,22]$. The few available longitudinal studies are generally consistent with cross-sectional results, and found positive associations between baseline/change in the social environment and change in PA. Much of the literature, especially on social capital, captures total PA or leisure-based 
PA and does not explore whether a specific aspect of social context could differentially affect a range of forms of PA, such as outside play, structured activities or walking to school. There is also limited evidence as to whether the positive associations observed for the general population are consistent among ethnic minority and deprived populations.

In this paper, we use data from the Olympic Regeneration in East London (ORiEL) study to explore how neighbourhood trust and social support from family, friends and significant others are longitudinally associated with four common forms of PA in a relatively deprived and ethnically diverse adolescent population. As Olympic-related regeneration accelerated ongoing transformations of East London, changes in the social environment are expected to have occurred before and during the ORiEL study period [23]. The ORiEL study therefore allows testing of hypotheses on 1) general associations between the social environment and PA; and 2) how short term changes in the social environment could immediately affect PA [17]. The PA outcomes we consider are walking to school, walking for leisure, outdoor PA and a composite measure of 'pay and play' PA. We additionally examine whether gender moderated these associations.

\section{Methods}

\section{Study design and participants}

We used data from the ORiEL study, a prospective cohort study that aimed to assess the effect of urban regeneration following the London 2012 Olympic and Paralympic Games on health. Participants were enrolled from 25 schools in four boroughs of London: Hackney, Newham, Barking and Dagenham, and Tower Hamlets. These boroughs are characterised by the high ethnic diversity of their populations and have higher levels of social, environmental and economic deprivation than the English and London average [24]. Schools were selected using simple randomisation with refusals replaced by eligible schools from the same borough. More information on the data collection and study recruitment is detailed elsewhere [24].

The participants were in Year 7 of school at baseline (age 11-12 years; January to June 2012). They were followed-up for a first time in Year 8 of school (wave 2: age 12-13 years; January to June 2013) and for a second time in Year 9 of school (wave 3: age 13-14 years; January to June 2014). To reduce seasonality effects, timing of follow-up was matched by month for each school. Because the exposure variables (neighbourhood trust and social support) were not available at baseline, we restricted the analyses to adolescents who participated at wave 2 and wave 3 . The final longitudinal cohort comprised all 2,664 adolescents of the 3,228 adolescents interviewed at wave 2, who were also followed-up at wave 3 (retention rate $82 \%$ ). 
This analysis was conducted in accordance with the guidelines dictated in the Declaration of Helsinki. The project obtained ethical approval from the Queen Mary University of London Research Ethics Committee (QMREC2011/40), the London Boroughs Research Governance Framework (CERGF113), and the Association of Directors of Children's Services (RGE110927). Head teachers provided written consent for the study to take place within their school, parents provided passive informed consent for their child to participate, and adolescent participants gave written informed assent.

\section{Measures}

\section{Exposures}

Four exposure variables were used to capture respondent perceptions of their social environment: neighbourhood trust, as well as social support from friends, family and significant others. Neighbourhood trust is a single-item obtained from a broader set of age-adapted questions on trust in different groups of people [25]. The question asks whether the respondents 'trust people in [their]

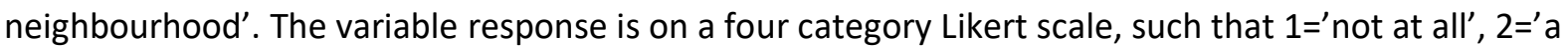
little', 3='some', 4='a lot'. The neighbourhood trust item was selected because it was expected to be particularly affected by Olympic-related regeneration.

The social support measures are derived from the 12-item Multidimensional Scale of Perceived Social Support (MSPSS) [26]. It is a composite measure of social support which is non-specific to PA and captures more emotional than instrumental forms of support. MSPSS items were rated on seven-point Likert scale ranging from 'agree very strongly' to 'disagree very strongly'. We summed scores for each source of support (i.e. friends, family and significant others) and split them into tertiles ( $1=^{\prime}$ low' $^{\prime}$ 2='medium', 3='high'), owing to their skewed positive distribution. Ordinal exposure variables were treated as either discrete or continuous when there was indication of a dose-response relationship. In addition, change scores in each exposure variable were calculated as the difference between the two data collection points in the numeric values to which time-varying exposure variable is coded. Positive scores indicate improvement in the exposure variables over time.

\section{PA outcomes}

PA was measured using the Youth PA Questionnaire (Y-PAQ). Y-PAQ is a validated self-reported instrument that assesses the duration and frequency of a series of PA and sedentary activities over the past 7 days [27]. We computed four forms of PA expected to be differentially associated with the exposure variables: walking to school, walking for leisure, outdoor PA and pay and play PA. Outdoor PA aims to group physical activities that are mainly performed in open recreation areas such as 
parks, sport fields and other open spaces, and which are usually located in the residential neighbourhood of adolescents [28,29]. The measure is composed of football, rounders, basketball/volleyball (mainly outdoor), cricket, rollerblading, roller skating and rugby. Pay and play PA captures scheduled formal PA, usually performed in sport or leisure centres and for which adolescents might need to pay in order to participate. It includes aerobics, climbing, swimming, gymnastics, hockey, martial arts, netball and tennis. Owing to the skewed distribution of the total time spent on each form of PA and to the fact that no adequate transformation could be found, the four outcome variables were dichotomised as 'activity reported at least once' vs. not $[30,31]$.

Measures of within individual change in the outcomes over time were also created and constructed as differences in the binary PA status between the two measurement points, resulting in ordinal variables with 3 responses categories $(0=$ stopped reporting the PA outcome at wave $3 ; 1=$ no change; $2=$ started reporting the PA outcome at wave 3 ).

\section{Covariates}

We identified potential confounders a priori from existing literature. Those available at both measurement points (wave 2 and wave 3 ) were added to the adjusted models if we found evidence of association with the exposures and PA. The adjusted models included: gender, ethnicity (White UK, White Mixed, Indian, Pakistani, Bangladeshi, Black Caribbean, Black African, or Other), household composition (both parents vs. not), time lived in the neighbourhood ( $\leq 5$ years vs. $>5$ ); free-school meal status, family affluence (3 categories from the revised Family Affluence Scale II), and the presence of health condition (none vs. 1+; from the following conditions: mobility problems, longstanding illness, anaemia, asthma, diabetes, Chronic Fatigue Syndrome, hay fever, hearing and eyesight problems) $[30,31]$.

\section{Statistical analyses}

Missing values ranged from $0.0 \%$ to $21.0 \%$. We investigated both the predictors of partially observed variables and the predictors of the probability of missingness using logistic regressions. These analyses indicated that the missing at random assumption was plausible. We used multilevel multiple imputation to impute missing data using the 'jomo' package in $R$ (based on a joint multivariate normal modelling approach) [32]. Auxiliary variables were total physical activity (logtransformed), country of birth, language spoken at home, mental health (squared WEMWBS score), BMI z-score, self-rated health, parental involvement, and neighbourhood satisfaction. Our imputation model included two levels (adolescents nested in schools). The outcomes and covariates were included as fixed effects, so that a separate variable was used for each measurement occasion. 
We handled interaction terms between the exposure variables and gender by imputing the data for each gender separately $[30,31]$. The imputation model was compatible with the most saturated model of analysis [33]. We used a 'burn in' period of 4,050 iterations for boys and girls, and 1,000 between-imputation iterations to generate 20 imputed datasets. The convergence of the Markov Chain Monte Carlo chains was satisfactory.

To assess general associations between exposures and the outcomes, we estimated unadjusted and adjusted logistic regression models with GEE (pooled models based on waves 2 and 3 ). This way of exploring associations provides information on whether differences in exposures, coming either from two different individuals or the same individual at different measurement points, lead to differences in PA. This modelling strategy is useful to capture evidence of association in situations with few repeated measurements or if exposures do not change much over time. We used GEE methods to account for the hierarchical structure of the data (although restricted to two levels of analysis), while preserving a population-average interpretation of the parameters [34]. The models accounted for the clustering due to repeated measurements on the same individuals (using unstructured working correlation structures). We did not adjust for all exposure variables together, given multicollinearity between the three sources of social support.

We then explored whether within individual changes over time in exposure were associated with changes in outcomes using proportional odds models. Adjusted models included confounders as measured at the first measurement point (wave 2). None of the models accounted for clustering at school-level as it was negligible for our PA outcomes [30].

Finally, we explored whether gender was a moderator by running a series of fully adjusted models that included an interaction term between each exposure of interest and gender. Stratum-specific results were reported for the interactions with $p$-values $<0.1$. Analyses were conducted using Stata 15.

\section{Results}

Walking to school and outdoor PA were most prevalent (respectively, $77 \%$ and $76 \%$ at wave 2 ), and walking for leisure least prevalent (35\% at wave 2) (Table 1). PA prevalence declined over time. While walking to school remained constant, walking for leisure and outdoor PA each decreased by about 5 percentage points at wave 3 and pay and play PA decreased by 13 percentage points. 
A majority of participants had at least some trust in their neighbours at each wave and high or medium levels of perceived social support from friends, family and significant others. While measures of social support had relatively stable distributions, those reporting no trust in their neighbours slightly increased at wave 3 (from 9.4\% to $12.4 \%$ ). The key socio-demographic characteristics of the sample are provided in Table 1. Overall, the sample was relatively deprived (37.3\% received free school meals at wave $2 ; 40.7 \%$ had low family affluence) and ethnically diverse (16.6\% were White UK and $36.3 \%$ were classified as Other).

\section{Walking to school}

The pooled models do not provide evidence of an association between neighbourhood trust and social support and walking to school (Table 2). Models of change in the exposures and outcomes confirm the absence of associations with walking to school (Table 3). The inclusion of interaction terms between each measure of the social environment and gender provides no evidence that gender moderates the associations, except for change in social support from significant others (Tables 2 and 3). For girls, there is weak evidence that improved social support from significant others over time might increase the odds of walking to school at wave $3(\mathrm{OR}=1.15 ; 95 \% \mathrm{Cl}$ : 0.98 1.35) (Table 4). 


\section{Walking for leisure}

The pooled model indicates that measures of social support, unlike neighbourhood trust, are positively associated with walking for leisure (Table 2). The evidence appears to be stronger for social support from family (OR trend=1.15; $95 \% \mathrm{Cl}: 1.06-1.25)$. The model for change in the exposure and outcomes however only provides evidence that increased social support from friends increases the odds of walking for leisure at wave 3 (OR=1.11; 95\% Cl: 1.01-1.22) (Table 3). Gender does not moderate these associations (Tables 2 and 3 ).

\section{Outdoor PA}

Results from the pooled model provide evidence of a positive dose-response association between neighbourhood trust and outdoor PA (OR trend=1.10; 95\% Cl: 1.01-1.19) (Table 2). Social support was not associated with outdoor PA in the general model, however, stratified analyses indicate evidence of a positive dose-response relationship between social support from friends and PA in boys (OR trend $=1.21 ; 95 \% \mathrm{Cl}: 1.04-1.42$ ) (Table 5). The models for changes in neighbourhood trust or social support indicate no evidence of associations with change in outdoor PA (Table 3).

\section{Pay and play PA}

As for outdoor PA there is strong evidence from the pooled model of a positive dose-response relationship between neighbourhood trust and pay and play PA (OR trend=1.09; 95\% $\mathrm{Cl}$ : 1.02-1.17), but no evidence for social support (Table 2). The models for changes in neighbourhood trust or social support indicate no evidence of associations with change in pay and play PA (Table 3). Nonetheless, stratified results provide weak evidence that, for boys only, improved neighbourhood might improve the odds of pay and play PA at wave 3 (OR=1.13; 95\% CI: 1.00-1.29) (Table 4). 


\section{Discussion}

In this paper, we examined associations between two aspects of the social environment neighbourhood trust and social support - and four PA outcomes in a deprived and ethnically diverse adolescent population. We found that perceived neighbourhood trust was positively associated with outdoor PA and with pay and play PA. There was also consistent evidence for an association between social support and walking for leisure. We did not find any evidence that walking to school was associated with any of the exposures.

\section{Neighbourhood trust}

The few studies which have investigated the associations between trust, or other aspects of social capital, and PA in adolescents have found positive associations with total and recreational PA though there is heterogeneity in the way exposures are measured [10-13]. For example, a recent crossnational study found consistent associations between collective efficacy and associated with objectively-measured total PA in 9-11 year old children in twelve high income countries [14].

The findings reported here are the first to investigate associations between trust and different forms of PA in the UK. We find evidence that neighbourhood trust was generally positively associated with leisure-time PA: walking for leisure, outdoor PA, and pay and play PA. This is consistent with previous results based on total or recreational PA as outcomes. Two plausible mechanisms could explain this. First, neighbourhood trust might favour autonomy and outside play by reducing fear of crime and increasing informal social control. Second, higher neighbourhood trust might also be indicative of stronger and better-organised communities, which might provide more opportunities for structured and unstructured PA [4]. In contrast, we found no evidence of an association with utilitarian walking, captured using walking to school. The prevalence of walking to school, unlike other forms of PA, did not decrease during the study period. This suggests that walking to school might be a more acceptable and pragmatic activity for adolescents, which is therefore less likely to be affected by the social environment.

In this study, most of the evidence for an association between neighbourhood trust and PA comes from pooled longitudinal models, in which the association comes either from cross-sectional information or from within individual changes over time. Although, in the models focusing on within individual changes we found very limited evidence of associations. Interestingly, however, a model for boys indicated that an increase in neighbourhood trust over time was associated with an improvement in pay and play PA. This suggests that, despite the very short period of follow-up (1 
year between wave 2 and wave 3), improvement in neighbourhood trust might have positive and relatively immediate consequences for leisure-time PA.

\section{Social support}

We found consistent associations between the three sources of social support (friends, family and significant others) and walking for leisure, but no consistent associations with walking to school, outdoor PA, or pay and play PA. These results contrast somewhat with the literature on social support and PA in young people which report consistent positive associations [16,19-21]. There are several possible reasons for this. First, we did not include a measure of total PA, for which a positive association might be observed, given the direction of the coefficients observed for walking for leisure and outdoor PA. Second, the MSPSS is generic tool which does not address PA specifically [20] and appears to be better at capturing emotional aspects of social support [35]. Instrumental support, co-participation and modelling, which were all shown to be relevant aspects of social support for PA $[16,19,20]$, are not explicitly mentioned in the MSPSS instrument. Therefore, if adolescents were receiving non-emotional forms of support, it may be under-reported. In particular, more structured activities captured by pay and play PA typically require instrumental support from the parents, such as paying participation fees, buying equipment, and providing transportation [36]. The absence of an observed association might reflect the possibility that such aspects of social support are poorly captured by the MSPSS, and therefore might under-estimate associations between social support and PA investigated here.

The main finding was to provide evidence supporting longitudinal associations, especially for social support from family and friends. These results are consistent with the few studies that have investigated changes in social support and changes in PA [17,37-39]. These generally show that increasing or maintaining general social support and encouragement from parents and friends during adolescence matters for PA. That we are able to observe improvements in PA from positive changes in social support over a short period of time (one year), suggest that interventions targeting social support might be beneficial for PA.

We found some evidence that gender moderated observed associations for walking to school and for outdoor PA. In boys, we found strong evidence that higher social support from friends, and weak evidence that family social support, increases the odds of outdoor PA, and this association is consistent with the literature [16]. The fact that the association was only observed for boys is unexpected as boys and girls received similar amount of social support. One possible explanation is that the type of support received might differ for boys and girls and be more relevant to PA for boys (e.g. transportation, co-participation, encouragement). 


\section{Strengths and limitations of this study}

To our knowledge, this is one of the first large-scale analyses of the longitudinal associations between social capital and social support with four measures of adolescent PA in the UK. We use statistical methods that account for non-independence of observations and item non-response. The Y-PAQ questionnaire allowed for the study of four common types of PA, and thus enabled us to explore how different aspects of PA were associated with measures of the social environment. Additional advantages of this study were the ethnic diversity of the sample; the high response rate ( $87 \%$ at wave 1 ) and the high retention rate ( $82 \%$ between wave 2 and wave 3 ).

However, there are also some limitations. PA is self-reported and is subject to social desirability and recall biases [40]. In addition, the psychometric properties of neighbourhood trust, as a single-item, have not been established. The super-diversity of the ORiEL sample is both a strength and a weakness as over 200 ethnic categories were self-reported by respondents, which restricted the ability to examine ethnic-specific effects of the social environment. Although the ORiEL study is one of the few large longitudinal studies to explore the determinants of PA, its short period of follow-up for which data were available ( 1 year) may have limited the ability to find longitudinal associations. Nonetheless, the physical, economic and social transformation of East London occurring around the time of the 2012 Olympic and Paralympic Games are likely to have affected the aspects of the social environment investigated in this study [23]. It is therefore likely that the extent of change in exposure observed within a year of follow-up is larger than would naturally occur in other studies conducted in less dynamic urban settings. It should also be noted that due to age-dependent variations in (changes in) social support and trust as adolescent grow-up, our results should not be generalised to older adolescents. Finally, we were unable to assess causal relationships. Though the longitudinal design can help strengthen causal inference, reverse causality cannot be ruled out.

\section{Conclusion}

Our findings suggest that different aspects of the social environment predict different types of adolescent PA. This suggests that there may be no 'one-size fits all' strategy to improving the social environment to increase adolescent PA. Policymakers and practitioners rather need to consider tailoring social support and social capital interventions in order to increase different forms of PA. 


\section{Declarations}

\section{Funding}

NB was supported by the Economic and Social Research Council (Grant No. 1482460). The ORiEL study was funded by the NIHR Public Health Research Programme (Grant No. 09/3005/09 to SC). The funding source had no role in the writing of this article nor the decision to submit it for publication.

\section{Availability of data and materials}

Restrictions apply to the availability of these data, which were used under license for the current study, and so are not publicly available. Data are however available from the authors upon reasonable request. Please contact SC with data requests.

\section{Author contributions}

NB conceived of the study, designed and executed the statistical analyses, and drafted the manuscript. MQ and ENN advised the statistical analyses and contributed to the interpretation of data. DL and SC supervised all aspects of data processing and analysis, and guided the design of this study. All authors critically revised the manuscript. All authors read and approved the final manuscript.

\section{Competing interests}

The authors declare that they have no competing interests. 


\section{References}

1 Brodersen NH, Steptoe A, Boniface DR, et al. Trends in physical activity and sedentary behaviour in adolescence: Ethnic and socioeconomic differences. Br J Sports Med 2007;41:140-4. doi:10.1136/bjsm.2006.031138

2 Health and Social Care Information Centre. Statistics on obesity, physical activity and diet : England 2017. 2017.

https://www.gov.uk/government/uploads/system/uploads/attachment_data/file/613532/ob es-phys-acti-diet-eng-2017-rep.pdf

3 Papas MA, Alberg AJ, Ewing R, et al. The built environment and obesity. Epidemiol Rev 2007;29:129-43.

4 Kawachi I, Berkman LF. Social cohesion, social capital, and health. In: Berkman LF, Kawachi I, Glymour MM, eds. Social Epidemiology. Oxford University Press 2014. 290-319.

5 Barnett E, Casper M. A definition of 'social environment'. Am J Public Health 2001;91:465.http://www.ncbi.nlm.nih.gov/pmc/articles/PMC1446600/

6 Berkman LF. Social Support, Social Networks, Social Cohesion and Health. Soc Work Health Care 2000;31:3-14. doi:10.1300/J010v31n02_02

7 Putnam RD. The prosperous community. Am Prospect 1993;4:35-42.

8 McNeill LH, Kreuter MW, Subramanian S V. Social environment and physical activity: a review of concepts and evidence. Soc Sci Med 2006;63:1011-22.

9 Lindström M. Social capital and health-related behaviors. In: Kawachi I, Kim D, eds. Social Capital and Health. Springer 2008. 215-38.

10 Carroll-Scott A, Gilstad-Hayden K, Rosenthal L, et al. Disentangling neighborhood contextual associations with child body mass index, diet, and physical activity: the role of built, socioeconomic, and social environments. Soc Sci Med 2013;95:106-14. doi:http://dx.doi.org/10.1016/j.socscimed.2013.04.003

11 Cradock AL, Kawachi I, Colditz GA, et al. Neighborhood social cohesion and youth participation in physical activity in Chicago. Soc Sci Med 2009;68:427-35. doi:http://dx.doi.org/10.1016/j.socscimed.2008.10.028

12 Franzini L, Elliott MN, Cuccaro P, et al. Influences of physical and social neighborhood 
environments on children's physical activity and obesity. Am J Public Health 2009;99:271-8. doi:10.2105/AJPH.2007.128702

Kimbro RT, Brooks-Gunn J, McLanahan S. Young children in urban areas: Links among neighborhood characteristics, weight status, outdoor play, and television watching. Soc Sci Med 2011;72:668-76. doi:10.1016/J.SOCSCIMED.2010.12.015

14 Sullivan SM, Broyles ST, Barreira T V, et al. Associations of neighborhood social environment attributes and physical activity among 9-11 year old children from 12 countries. Health Place 2017;46:183-91. doi:10.1016/j.healthplace.2017.05.013 Langford CP, Bowsher J, Maloney JP, et al. Social support: a conceptual analysis. J Adv Nurs 1997;25:95-100.http://www.ncbi.nlm.nih.gov/pubmed/9004016 (accessed 4 Dec 2017). systematic review. Health Educ Res 2014;29:82239.http://her.oxfordjournals.org/content/early/2014/05/07/her.cyu017.short (accessed 18 May 2017).

17 Lau EY, Faulkner G, Qian W, et al. Longitudinal associations of parental and peer influences with physical activity during adolescence: findings from the COMPASS study. Heal Promot Chronic Dis Prev Canada Res Policy Pract 2016;36:23542.http://www.ncbi.nlm.nih.gov/pubmed/27882858 (accessed 12 Jan 2018). Sallis JF, Bull F, Guthold R, et al. Progress in physical activity over the Olympic quadrennium. Lancet 2016;388:1325-36. doi:10.1016/S0140-6736(16)30581-5

19 Laird Y, Fawkner S, Kelly P, et al. The role of social support on physical activity behaviour in adolescent girls: a systematic review and meta-analysis. Int J Behav Nutr Phys Act 2016;13:79. doi:10.1186/s12966-016-0405-7

Beets MW, Cardinal BJ, Alderman BL. Parental social support and the physical activity-related behaviors of youth: a review. Heal Educ Behav 2010;37:621-44. doi:10.1177/1090198110363884

21 Yao CA, Rhodes RE. Parental correlates in child and adolescent physical activity: a metaanalysis. Int J Behav Nutr 2015;12:10. doi:10.1186/s12966-015-0163-y

22 Carrillo-Álvarez E, Kawachi I, Riera-Romaní J. Neighbourhood social capital and obesity: a systematic review of the literature. Obes Rev 2019;20:119-41. doi:10.1111/obr.12760 
23 Cummins S, Clark C, Lewis DJ, et al. Evaluating the impact of Olympic-related urban regeneration on physical activity and psychological health and wellbeing in adolescents and their parents: The ORiEL Study. 2017.

24 Smith NR, Clark C, Fahy AE, et al. The Olympic Regeneration in East London (ORiEL) study: protocol for a prospective controlled quasi-experiment to evaluate the impact of urban regeneration on young people and their families. BMJ Open 2012;2:e001840. doi:10.1136/bmjopen-2012-001840

252000 Social Capital Community Benchmark Survey. https://ropercenter.cornell.edu/featuredcollections/2000-social-capital-community-benchmark-survey

26 Zimet GD, Powell SS, Farley GK, et al. Psychometric characteristics of the Multidimensional Scale of Perceived Social Support. J Pers Assess 1990;55:610-7. doi:10.1080/00223891.1990.9674095

27 Corder K, van Sluijs EM, Wright A, et al. Is it possible to assess free-living physical activity and energy expenditure in young people by self-report? Am J Clin Nutr 2009;89:862-70. doi:10.3945/ajcn.2008.26739

28 D'Haese S, Van Dyck D, De Bourdeaudhuij I, et al. The association between the parental perception of the physical neighborhood environment and children's location-specific physical activity. BMC Public Health 2015;15:565. doi:10.1186/s12889-015-1937-5

29 Esteban-Cornejo I, Carlson JA, Conway TL, et al. Parental and adolescent perceptions of neighborhood safety related to adolescents' physical activity in their neighborhood. Res $Q$ Exerc Sport 2016;87:191-9. doi:10.1080/02701367.2016.1153779

30 Berger N, Lewis D, Quartagno M, et al. Associations between school and neighbourhood ethnic density and physical activity in adolescents: Evidence from the Olympic Regeneration in East London (ORiEL) study. Soc Sci Med 2019;:112426. doi:10.1016/J.SOCSCIMED.2019.112426

31 Berger N, Lewis D, Quartagno $M$, et al. Longitudinal associations between perceptions of the neighbourhood environment and physical activity in adolescents: Evidence from the Olympic Regeneration in East London (ORiEL) study. BMC Public Health 2019;19:1760. doi:10.1186/s12889-019-8003-7

32 Quartagno M, Grund S, Carpenter J. jomo: A Flexible Package for Two-level Joint Modelling Multiple Imputation. $R$ J 2019;9:1. 
34 Fitzmaurice GM, Laird NM, Ware JH. Applied longitudinal analysis. Wiley 2011.

35 Dahlem NW, Zimet GD, Walker RR. The Multidimensional Scale of Perceived Social Support: A confirmation study. J Clin Psychol 1991;47:756-61. doi:10.1002/10974679(199111)47:6<756::AID-JCLP2270470605>3.0.CO;2-L

Edwardson CL, Gorely T. Parental influences on different types and intensities of physical activity in youth: a systematic review. Psychol Sport Exerc 2010;11:522-35. doi:10.1016/J.PSYCHSPORT.2010.05.001

37 Zook KR, Saksvig BI, Wu TT, et al. Physical activity trajectories and multilevel factors among adolescent girls. J Adolesc Heal 2014;54:74-80. doi:10.1016/j.jadohealth.2013.07.015

38 Dowda M, Dishman RK, Pfeiffer KA, et al. Family support for physical activity in girls from 8th to 12th grade in South Carolina. Prev Med 2007;44:153-9. doi:10.1016/J.YPMED.2006.10.001 Davison KK, Jago R. Change in parent and peer support across ages 9 to $15 \mathrm{yr}$ and adolescent girls' physical activity. Med Sci Sport Exerc 2009;41:1816-25.

doi:10.1249/MSS.0b013e3181a278e2 assessing physical activity in adults: a systematic review. Int J Behav Nutr Phys Act 2008;5:56. doi:10.1186/1479-5868-5-56 
Table 1 Characteristics of the ORiEL study participants, 2013-2014 $(n=2,644)$

\begin{tabular}{|c|c|c|c|}
\hline & 2013 & 2014 & $\%$ \\
\hline & & & Missing \\
\hline \multicolumn{4}{|l|}{ Exposures } \\
\hline Neighbourhood trust & & & 13.9 \\
\hline Not at all & 9.4 & 12.4 & \\
\hline A little & 27.7 & 27.6 & \\
\hline Some & 41.4 & 42.4 & \\
\hline A lot & 21.5 & 17.6 & \\
\hline Social support - friends & & & 20.7 \\
\hline Low & 39.4 & 40.6 & \\
\hline Medium & 28.4 & 31.2 & \\
\hline High & 32.2 & 28.2 & \\
\hline Social support - family & & & 20.4 \\
\hline Low & 29.6 & 33.4 & \\
\hline Medium & 27.3 & 30.1 & \\
\hline High & 43.1 & 36.5 & \\
\hline Social support - significant others & & & 21.0 \\
\hline Low & 40.7 & 40.9 & \\
\hline Medium & 26.9 & 28.0 & \\
\hline High & 32.4 & 31.1 & \\
\hline \multicolumn{4}{|l|}{ Outcomes } \\
\hline$\%$ walking to school & 76.6 & 75.7 & 3.5 \\
\hline$\%$ walking for leisure & 35.0 & 30.1 & 6.1 \\
\hline$\%$ reporting outdoor $\mathrm{PA}$ & 76.0 & 71.0 & 10.4 \\
\hline$\%$ reporting pay and play PA & 64.1 & 51.3 & 9.5 \\
\hline \multicolumn{4}{|l|}{ Covariates } \\
\hline$\%$ Girls & 56.7 & - & 0.0 \\
\hline Ethnicity & & & 0.1 \\
\hline$\%$ White: UK & 16.6 & - & \\
\hline$\%$ White: Mixed & 8.3 & - & \\
\hline
\end{tabular}




\begin{tabular}{llll} 
\% Asian: Indian & 3.7 & - \\
\% Asian: Pakistani & 3.9 & - & \\
\% Asian: Bangladeshi & 15.1 & - & \\
\% Black: Caribbean & 4.9 & - & \\
\% Black: African & 11.2 & - & \\
\% Other & 36.3 & - & 14.4 \\
\% with health condition & 39.7 & 41.4 & 1.8 \\
\% receiving free school meals & 37.3 & 32.5 & 3.6 \\
Family affluence & & & \\
\% Low & & 4.9 & \\
\% Medium & 7.1 & 51.5 & 0.8 \\
\% High & 50.6 & 43.6 & 4.9 \\
\% not living with both parents & 31.4 & 32.9 & 63.1 \\
\% living in the neighbourhood $>5$ y & 60.7 & & \\
\hline
\end{tabular}


Table 2 General associations of the social environment with physical activity $(n=2,644)$

\begin{tabular}{|c|c|c|c|c|c|c|c|c|}
\hline \multirow[b]{2}{*}{ Exposure } & & \multicolumn{3}{|c|}{ Unadjusted } & \multicolumn{3}{|c|}{ Adjusted $^{1}$} & \multirow{2}{*}{\begin{tabular}{|l|} 
Gender-int. $^{2}$ \\
p-value
\end{tabular}} \\
\hline & & OR & $95 \% \mathrm{Cl}$ & p-value & OR & $95 \% \mathrm{Cl}$ & p-value & \\
\hline \multicolumn{9}{|c|}{ Outcome: Walking to school } \\
\hline \multirow[t]{4}{*}{ Neighbour. trust } & Not at all & 1.00 & & 0.296 & 1.00 & & 0.482 & 0.528 \\
\hline & A little & 1.02 & {$[0.80,1.30]$} & & 0.99 & {$[0.77,1.26]$} & & \\
\hline & Some & 1.17 & {$[0.93,1.47]$} & & 1.10 & {$[0.88,1.39]$} & & \\
\hline & A lot & 1.06 & {$[0.81,1.38]$} & & 0.98 & {$[0.75,1.28]$} & & \\
\hline \multirow[t]{3}{*}{ Soc. sup. - friends } & Low & 1.00 & & 0.253 & 1.00 & & 0.170 & 0.258 \\
\hline & Medium & 1.10 & {$[0.93,1.29]$} & & 1.07 & {$[0.91,1.27]$} & & \\
\hline & High & 0.95 & {$[0.81,1.12]$} & & 0.90 & {$[0.76,1.07]$} & & \\
\hline \multirow[t]{3}{*}{ Soc. sup. - family } & Low & 1.00 & & 0.753 & 1.00 & & 0.680 & 0.062 \\
\hline & Medium & 0.95 & {$[0.80,1.14]$} & & 0.95 & {$[0.79,1.15]$} & & \\
\hline & High & 0.94 & {$[0.79,1.11]$} & & 0.93 & {$[0.78,1.10]$} & & \\
\hline \multirow[t]{3}{*}{ Soc. sup. - sig. oth. } & Low & 1.00 & & 0.916 & 1.00 & & 0.934 & 0.265 \\
\hline & Medium & 0.97 & {$[0.81,1.15]$} & & 0.97 & {$[0.81,1.16]$} & & \\
\hline & High & 1.00 & {$[0.85,1.17]$} & & 0.98 & {$[0.83,1.16]$} & & \\
\hline \multicolumn{9}{|c|}{ Outcome: Walking for leisure } \\
\hline \multirow[t]{5}{*}{ Neighbour. trust } & Not at all & 1.00 & & 0.193 & 1.00 & & 0.314 & 0.919 \\
\hline & A little & 1.28 & {$[1.02,1.61]$} & & 1.24 & {$[0.98,1.57]$} & & \\
\hline & Some & 1.25 & {$[1.00,1.56]$} & & 1.24 & {$[0.99,1.55]$} & & \\
\hline & A lot & 1.20 & {$[0.94,1.52]$} & & 1.22 & {$[0.95,1.57]$} & & \\
\hline & Trend $^{5}$ & 1.03 & {$[0.96,1.11]$} & 0.414 & 1.04 & {$[0.97,1.12]$} & 0.250 & 0.822 \\
\hline \multirow[t]{4}{*}{ Soc. sup. - friends } & Low & 1.00 & & 0.001 & 1.00 & & 0.079 & 0.464 \\
\hline & Medium & 1.24 & {$[1.06,1.44]$} & & 1.17 & {$[1.00,1.37]$} & & \\
\hline & High & 1.31 & {$[1.12,1.53]$} & & 1.17 & {$[0.99,1.38]$} & & \\
\hline & Trend $^{5}$ & 1.15 & {$[1.06,1.24]$} & 0.001 & 1.08 & {$[1.00,1.18]$} & 0.050 & 0.205 \\
\hline \multirow[t]{4}{*}{ Soc. sup. - family } & Low & 1.00 & & $<0.001$ & 1.00 & & 0.004 & 0.641 \\
\hline & Medium & 1.20 & {$[1.01,1.43]$} & & 1.19 & {$[1.00,1.42]$} & & \\
\hline & High & 1.38 & {$[1.18,1.62]$} & & 1.32 & {$[1.12,1.56]$} & & \\
\hline & Trend $^{5}$ & 1.17 & {$[1.08,1.27]$} & $<0.001$ & 1.15 & {$[1.06,1.25]$} & 0.001 & 0.352 \\
\hline \multirow[t]{4}{*}{ Soc. sup. - sig. oth. } & Low & 1.00 & {$[0.95,1.27]$} & 0.001 & 1.00 & & 0.055 & 0.474 \\
\hline & Medium & 1.18 & {$[1.02,1.38]$} & & 1.11 & {$[0.95,1.30]$} & & \\
\hline & High & 1.34 & {$[1.14,1.56]$} & & 1.21 & {$[1.03,1.43]$} & & \\
\hline & Trend $^{5}$ & 1.16 & {$[1.07,1.25]$} & $<0.001$ & 1.10 & {$[1.02,1.20]$} & 0.019 & 0.373 \\
\hline \multicolumn{9}{|c|}{ Outcome: Outdoor $\mathrm{PA}^{3}$} \\
\hline \multirow[t]{5}{*}{ Neighbour. trust } & Not at all & 1.00 & & $<0.001$ & 1.00 & & 0.099 & 0.680 \\
\hline & A little & 1.03 & {$[0.82,1.28]$} & & 0.97 & {$[0.76,1.24]$} & & \\
\hline & Some & 1.16 & {$[0.95,1.43]$} & & 1.08 & {$[0.86,1.36]$} & & \\
\hline & A lot & 1.60 & {$[1.24,2.05]$} & & 1.29 & {$[0.97,1.70]$} & & \\
\hline & Trend 5 & 1.17 & {$[1.09,1.26]$} & $<0.001$ & 1.10 & {$[1.01,1.19]$} & 0.029 & 0.390 \\
\hline
\end{tabular}




\begin{tabular}{|c|c|c|c|c|c|c|c|c|}
\hline \multirow[b]{2}{*}{ Exposure } & & \multicolumn{3}{|c|}{ Unadjusted } & \multicolumn{3}{|c|}{ Adjusted $^{1}$} & \multirow{2}{*}{$\begin{array}{l}\text { Gender-int. }{ }^{2} \\
\text {-value }\end{array}$} \\
\hline & & $\overline{\text { OR }}$ & $95 \% \mathrm{Cl}$ & p-value & OR & $95 \% \mathrm{Cl}$ & $p$-value & \\
\hline \multirow[t]{3}{*}{ Soc. sup. - friends } & Low & 1.00 & & 0.164 & 1.00 & & 0.748 & 0.027 \\
\hline & Medium & 0.91 & {$[0.77,1.07]$} & & 1.06 & {$[0.89,1.27]$} & & \\
\hline & High & 0.86 & {$[0.73,1.01]$} & & 1.06 & {$[0.89,1.26]$} & & \\
\hline \multirow[t]{3}{*}{ Soc. sup. - family } & Low & 1.00 & & 0.844 & 1.00 & & 0.815 & 0.179 \\
\hline & Medium & 1.01 & {$[0.85,1.20]$} & & 1.05 & {$[0.88,1.26]$} & & \\
\hline & High & 1.04 & {$[0.89,1.22]$} & & 1.05 & {$[0.88,1.25]$} & & \\
\hline \multirow[t]{3}{*}{ Soc. sup. - sig. oth. } & Low & 1.00 & & 0.273 & 1.00 & & 0.881 & 0.354 \\
\hline & Medium & 0.90 & {$[0.76,1.06]$} & & 1.03 & {$[0.86,1.23]$} & & \\
\hline & High & 0.89 & {$[0.76,1.03]$} & & 1.04 & {$[0.89,1.23]$} & & \\
\hline \multicolumn{9}{|c|}{ Outcome: Pay and play PA ${ }^{4}$} \\
\hline \multirow[t]{5}{*}{ Neighbour. trust } & Not at all & 1.00 & & 0.005 & 1.00 & & 0.025 & 0.695 \\
\hline & A little & 1.03 & {$[0.82,1.29]$} & & 0.95 & {$[0.75,1.20]$} & & \\
\hline & Some & 1.10 & {$[0.89,1.35]$} & & 1.03 & {$[0.82,1.27]$} & & \\
\hline & A lot & 1.40 & {$[1.10,1.78]$} & & 1.27 & {$[0.99,1.63]$} & & \\
\hline & Trend $^{5}$ & 1.12 & {$[1.04,1.20]$} & 0.001 & 1.09 & {$[1.02,1.17]$} & 0.013 & 0.850 \\
\hline \multirow[t]{3}{*}{ Soc. sup. - friends } & Low & 1.00 & & 0.975 & 1.00 & & 0.891 & 0.528 \\
\hline & Medium & 1.00 & {$[0.86,1.15]$} & & 1.00 & {$[0.86,1.17]$} & & \\
\hline & High & 1.01 & {$[0.87,1.18]$} & & 0.97 & {$[0.83,1.13]$} & & \\
\hline \multirow[t]{3}{*}{ Soc. sup. - family } & Low & 1.00 & & 0.624 & 1.00 & & 0.968 & 0.470 \\
\hline & Medium & 1.00 & {$[0.86,1.17]$} & & 0.98 & {$[0.84,1.15]$} & & \\
\hline & High & 1.07 & {$[0.91,1.26]$} & & 0.98 & {$[0.83,1.16]$} & & \\
\hline \multirow[t]{3}{*}{ Soc. sup. - sig. oth. } & Low & 1.00 & & 0.761 & 1.00 & & 0.867 & 0.847 \\
\hline & Medium & 0.99 & {$[0.85,1.16]$} & & 0.96 & {$[0.82,1.13]$} & & \\
\hline & High & 1.05 & {$[0.90,1.22]$} & & 1.00 & {$[0.85,1.17]$} & & \\
\hline
\end{tabular}

Results are from logistic regression models estimated with Generalised Estimating Equations to account for the dependency across repeated measurements (unstructured working correlation matrix).

${ }^{1}$ Adjusted for gender, ethnicity, health condition, free school meal status, family affluence, time lived in the neighbourhood, household composition and time.

${ }^{2}$ The adjusted model was replicated for each outcome with an additional interaction term between gender and exposure.

${ }^{3}$ Outdoor PA include: basketball (or volleyball), blading, cricket, football, rounders, rugby and roller skating.

${ }^{4}$ Pay and play PA include: aerobics, climbing, swimming, gymnastics, hockey, martial arts, netball, and tennis.

${ }^{5}$ Exposure modelled as a continuous variable when indication of a dose-response relationship.

OR - Odds ratio, int. - interaction, Neighbour. - Neighbourhood, Soc. sup. - Social support, sig. oth. - significant others, 
Table 3 Associations of change in the social environment with change in physical activity $(n=2,664)$

\begin{tabular}{|c|c|c|c|c|c|c|c|}
\hline \multirow[b]{2}{*}{ Exposure } & \multicolumn{3}{|c|}{ Unadjusted } & \multicolumn{3}{|c|}{ Adjusted $^{1}$} & \multirow{2}{*}{\begin{tabular}{|l} 
Gender-interaction ${ }^{2}$ \\
p-value
\end{tabular}} \\
\hline & OR & $95 \% \mathrm{Cl}$ & p-value & OR & $95 \% \mathrm{Cl}$ & p-value & \\
\hline \multicolumn{8}{|c|}{ Outcome: Walking to school } \\
\hline Neighbour. trust & 1.02 & {$[0.92,1.14]$} & 0.698 & 1.03 & {$[0.92,1.15]$} & 0.605 & 0.145 \\
\hline Soc. sup. - friends & 0.97 & {$[0.87,1.08]$} & 0.598 & 0.97 & {$[0.87,1.08]$} & 0.575 & 0.157 \\
\hline Soc. sup. - family & 1.01 & {$[0.90,1.14]$} & 0.831 & 1.00 & {$[0.89,1.13]$} & 0.956 & 0.529 \\
\hline Soc. sup. - sig. oth. & 1.03 & {$[0.92,1.14]$} & 0.618 & 1.02 & {$[0.92,1.14]$} & 0.694 & 0.071 \\
\hline
\end{tabular}

Outcome: Walking for leisure

\begin{tabular}{lll|ll|l}
\hline Neighbour. trust & $1.07[0.98,1.17]$ & 0.127 & $1.07[0.98,1.17]$ & 0.156 & 0.876 \\
Soc. sup. - friends & $1.11[1.01,1.22]$ & 0.031 & $1.11[1.01,1.22]$ & 0.037 & 0.447 \\
Soc. sup. - family & $1.07[0.97,1.19]$ & 0.189 & $1.07[0.97,1.19]$ & 0.189 & 0.489 \\
Soc. sup. - sig. oth. & $1.05[0.95,1.15]$ & 0.351 & $1.04[0.95,1.15]$ & 0.364 & 0.167 \\
\hline
\end{tabular}

Outcome: Outdoor PA

\begin{tabular}{lll|ll|l}
\hline Neighbour. trust & $0.99[0.90,1.09]$ & 0.847 & $0.99[0.90,1.09]$ & 0.834 & 0.641 \\
Soc. sup. - friends & $1.01[0.91,1.12]$ & 0.893 & $1.01[0.91,1.12]$ & 0.867 & 0.315 \\
Soc. sup. - family & $0.97[0.87,1.08]$ & 0.582 & $0.97[0.87,1.08]$ & 0.543 & 0.556 \\
Soc. sup. - sig. oth. & $1.01[0.91,1.12]$ & 0.875 & $1.00[0.90,1.12]$ & 0.947 & 0.785 \\
\hline
\end{tabular}

Outcome: Pay and play PA ${ }^{4}$

\begin{tabular}{lll|ll|l}
\hline Neighbour. trust & $1.06[0.97,1.16]$ & 0.170 & $1.06[0.97,1.16]$ & 0.201 & 0.099 \\
Soc. sup. - friends & $0.99[0.91,1.09]$ & 0.891 & $0.99[0.91,1.08]$ & 0.845 & 0.695 \\
Soc. sup. - family & $0.98[0.89,1.09]$ & 0.741 & $0.98[0.88,1.09]$ & 0.691 & 0.275 \\
Soc. sup. - sig. oth. & $0.98[0.89,1.08]$ & 0.672 & $0.98[0.89,1.08]$ & 0.672 & 0.340
\end{tabular}

Results are from proportional odds models. Results are displayed as ORs of change in PA status (contrasting increase vs. constant high/low or decrease; or increase or constant high/low vs. decrease) per unit increase in the original scale of neighbourhood trust or tertile change in social support. ORs $>1$ indicate a positive change in the outcome as a response to an improvement in the exposure.

${ }^{1}$ Adjusted for gender, ethnicity, health condition, free school meal status, family affluence, time lived in the neighbourhood and household composition at wave 2 .

${ }^{2}$ The adjusted model was replicated for each outcome with an additional interaction term between gender and exposure.

${ }^{3}$ Outdoor PA include: basketball (or volleyball), blading, cricket, football, rounders, rugby and roller skating.

${ }^{4}$ Pay and play PA include: aerobics, climbing, swimming, gymnastics, hockey, martial arts, netball, and tennis. OR - Odds ratio, Neighbour. - Neighbourhood, Soc. sup. - Social support, sig. oth. - significant others, 
Table 4 Associations of change in the social environment with change in walking to school and change in pay and play PA by gender $(n=2,664)$

\begin{tabular}{|c|c|c|c|c|c|c|}
\hline \multirow[b]{2}{*}{ Exposure } & \multicolumn{3}{|c|}{ Unadjusted } & \multicolumn{3}{|c|}{ Adjusted $^{1}$} \\
\hline & OR & $95 \% \mathrm{Cl}$ & p-value & OR & $95 \% \mathrm{Cl}$ & p-value \\
\hline \multicolumn{7}{|c|}{ Outcome: Walking to school } \\
\hline \multicolumn{7}{|l|}{ Soc. sup. - sig. oth. } \\
\hline Boys & 0.94 & {$[0.80,1.09]$} & 0.410 & 0.93 & {$[0.80,1$} & 0.369 \\
\hline Girls & 1.15 & {$[0.98,1.34]$} & 0.086 & 1.15 & {$[0.98,1$} & 0.091 \\
\hline
\end{tabular}

Outcome: Pay and play PA $^{2}$

\begin{tabular}{|c|c|c|c|}
\hline \multicolumn{4}{|c|}{ Neighbour. trust } \\
\hline Boys & $1.14[1.00,1.30]$ & 0.040 & $1.13[1.00,1.29] 0.055$ \\
\hline Girls & $0.97[0.85,1.11]$ & 0.665 & $0.98[0.86,1.11] 0.718$ \\
\hline
\end{tabular}

Results are from proportional odds models. Results are displayed as ORs of change in PA status (contrasting increase vs. constant high/low or decrease; or increase or constant high/low vs. decrease) per unit increase in the original scale of neighbourhood trust or tertile change in social support. ORs $>1$ indicate a positive change in the outcome as a response to an improvement in the exposure.

${ }^{1}$ Adjusted for gender, ethnicity, health condition, free school meal status, family affluence, time lived in the neighbourhood and household composition at wave 2 .

${ }^{2}$ Outdoor PA include: basketball (or volleyball), blading, cricket, football, rounders, rugby and roller skating.

OR - Odds ratio, Neighbour. - Neighbourhood, Soc. sup. - Social support, sig. oth. - significant others 
Table 5 General associations of the social environment with outdoor PA ${ }^{1}$ by gender $(n=2,644)$

\begin{tabular}{|c|c|c|c|c|c|c|c|}
\hline \multicolumn{2}{|l|}{ Exposure } & \multirow{2}{*}{$\begin{array}{c}\begin{array}{c}\text { Adjusted } \\
\mathrm{OR}^{2}\end{array} \\
\text { Boys } \\
\end{array}$} & \multirow[t]{2}{*}{$95 \% \mathrm{Cl}$} & \multirow[t]{2}{*}{$p$-value } & \multirow{2}{*}{$\begin{array}{c}\begin{array}{c}\text { Adjusted } \\
\text { OR }^{2}\end{array} \\
\text { Girls } \\
\end{array}$} & \multirow[t]{2}{*}{$95 \% \mathrm{Cl}$} & \multirow[t]{2}{*}{ p-value } \\
\hline & & & & & & & \\
\hline \multirow[t]{4}{*}{ Soc. sup. - friends } & Low & 1.00 & & 0.039 & 1.00 & & 0.509 \\
\hline & Medium & 1.22 & {$[0.92,1.61]$} & & 0.93 & {$[0.75,1.17]$} & \\
\hline & High & 1.47 & {$[1.07,2.02]$} & & 0.88 & {$[0.70,1.10]$} & \\
\hline & Trend $^{3}$ & 1.21 & {$[1.04,1.42]$} & 0.014 & - & - & - \\
\hline \multirow[t]{4}{*}{ Soc. sup. - family } & Low & 1.00 & & 0.166 & 1.00 & & 0.733 \\
\hline & Medium & 1.18 & {$[0.87,1.60]$} & & 0.98 & {$[0.77,1.24]$} & \\
\hline & High & 1.31 & {$[0.99,1.74]$} & & 0.92 & {$[0.74,1.15]$} & \\
\hline & Trend $^{3}$ & 1.15 & {$[0.99,1.32]$} & 0.060 & - & - & - \\
\hline \multirow[t]{4}{*}{ Soc. sup. - sig. oth. } & Low & 1.00 & & 0.266 & 1.00 & & 0.836 \\
\hline & Medium & 1.16 & {$[0.87,1.53]$} & & 0.94 & {$[0.74,1.19]$} & \\
\hline & High & 1.24 & {$[0.95,1.63]$} & & 0.94 & {$[0.75,1.17]$} & \\
\hline & Trend $^{3}$ & 1.12 & {$[0.98,1.28]$} & 0.106 & - & - & - \\
\hline
\end{tabular}

Results are from logistic regression models estimated with Generalised Estimating Equations to account for the dependency across repeated (unstructured working correlation matrix).

${ }^{1}$ Outdoor PAs include: basketball (or volleyball), blading, cricket, football, rounders, rugby and roller skating.

2 Adjusted for ethnicity, health condition, free school meal status, family affluence, time lived in the neighbourhood, household composition and time.

${ }^{3}$ Exposure modelled as a continuous variable when indication of a dose-response relationship.

OR - Odds ratio, Neighbour. - Neighbourhood, Soc. sup. - Social support, sig. oth. - significant others 